
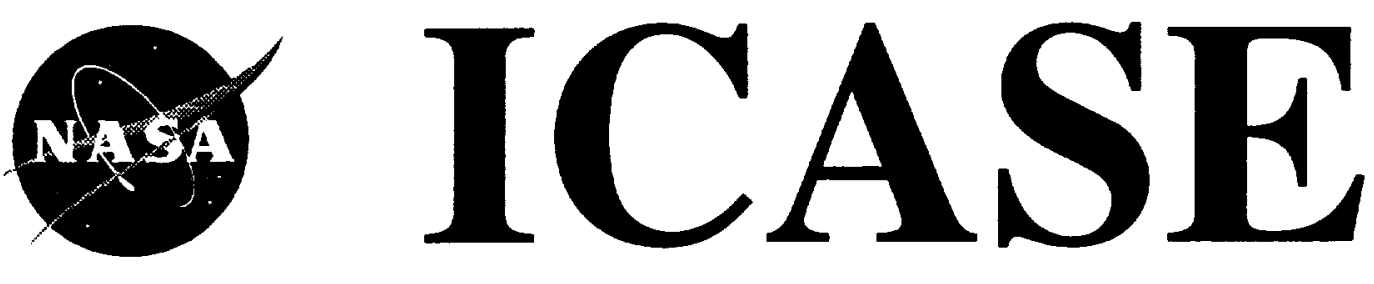

\title{
CONTRIBUTION TO THE OPTIMAL SHAPE DESIGN OF TWO-DIMENSIONAL INTERNAL FLOWS WITH EMBEDDED SHOCKS
}

Angelo Iollo Manuel D. Salas
(NASA-CR-195062) CONTRIBUTION TO

THE OPTIMAL SHAPE DESIGN OF

TWO-DIMENSIONAL INTERNAL FLOWS WITH EMBEDDED SHOCKS (ICASE) $23 \mathrm{P}$
N95-26672

Unclas

Contract No. NAS1-19480

March 1995

Institute for Computer Applications in Science and Engineering NASA Langley Research Center

Hampton, VA 23681-0001

SRA Operated by Universities Space Research Association 
. 


\title{
CONTRIBUTION TO THE OPTIMAL SHAPE DESIGN OF TWO-DIMENSIONAL INTERNAL FLOWS WITH EMBEDDED SHOCKS
}

\author{
Angelo Iollo ${ }^{1}$ \\ Dipartimento di Ingegneria Aeronautica e Spaziale \\ Politecnico di Torino \\ 10129 Torino, Italy \\ Manuel D. Salas \\ NASA Langley Research Center \\ Hampton, VA 23681-0001
}

\begin{abstract}
We explore the praticability of optimal shape design for flows modeled by the Euler equations. We define a functional whose minimum represents the optimality condition. The gradient of the functional with respect to the geometry is calculated with the Lagrange multipliers, which are determined by solving a costate equation. The optimization problem is then examined by comparing the performance of several gradient-based optimization algorithms. In this formulation, the flow field can be computed to an arbitrary order of accuracy. Finally, some results for internal flows with embedded shocks are presented, including a case for which the solution to the inverse problem does not belong to the design space.
\end{abstract}

\footnotetext{
${ }^{1}$ This research was supported in part under NASA contract no. NAS1-19480 while the author was in residence at the Institute for Computer Applications in Science and Engineering, NASA Langley Research Center, Hampton, VA 23681-0001.
} 



\section{Introduction}

A classical problem in engineering is to define the shape of a vehicle to achieve a required performance. In fluid dynamics, techniques have been developed to solve the following inverse problem: given a pressure or a velocity distribution over an aerodynamic body, determine the corresponding geometry. See, for example, reference [8]. A broader category of problems can be solved by means of optimization, provided that one is ready to accept the necessity of computing the flow field hundreds of times. In using models of increased complexity to describe the flow field, the development of new algorithms is necessary in many cases to reduce the computational load. In this paper, we investigate one method for achieving this reduction. The variational technique that is applied in this work has been used since before complex flows could be integrated numerically. See, for example, reference [1]. Jameson [4] was the first to apply this technique to computational fluid dynamics. With this approach, a functional or cost function is determined such that its minimum represents an optimal solution. By introducing a set of Lagrange multipliers, the gradient of the functional can be calculated with respect to the geometry by computing the flow field only once for each gradient evaluation. For incompressible irrotational steady flows, a further reduction in the computational effort is possible. (See [10].) The formulation developed in this work is more suited to a correct analysis. In fact, this formulation eliminates the need to consider the flow field variables dependent on the geometry. In the present work, we extend the work presented in reference [5]. In reference [5], an exact gradient of the functional with respect to the design variables was obtained on the discrete level, which can be a limitation for compressible flows because in presence of shocks the discrete functional can present discontinuities. In considering compressible flows with embedded shocks, we derive the gradient on a continuous level. Furthermore, we provide a method for calculating the conditions that the Lagrange multipliers must satisfy at the boundaries and at the shock. Finally, we point out that our formulation can be used with complex flow solvers because the differentiability of the solver is not requested.

\section{Problem Statement}

The Euler equations are given by

$$
\mathrm{U}_{t}+\mathbf{F}_{x}+\mathbf{G}_{y}=\mathbf{0}
$$

where

$$
\mathbf{U}=\left(\begin{array}{c}
\rho \\
\rho u \\
\rho v \\
\rho e
\end{array}\right) \quad \mathbf{F}=\left(\begin{array}{c}
\rho u \\
p+\rho u^{2} \\
\rho u v \\
u(\rho e+p)
\end{array}\right) \quad \mathbf{G}=\left(\begin{array}{c}
\rho v \\
\rho u v \\
p+\rho v^{2} \\
v(\rho e+p)
\end{array}\right)
$$

with

$$
\rho=\text { density }
$$




$$
\begin{aligned}
u & =x \text { component of velocity vector } \\
v & =y \text { component of velocity vector } \\
e & =\text { specific total energy } \\
p & =\text { pressure } \\
a & =\text { speed of sound } \\
\gamma & =\text { specific heats ratio } \\
\kappa & =\frac{\gamma-1}{2}
\end{aligned}
$$

and $p=\kappa \rho\left(2 e-u^{2}-v^{2}\right)$. Furthermore,

$$
\mathbf{F}=\frac{\partial \mathbf{F}}{\partial \mathbf{U}} \mathbf{U}=\mathbf{A}(\mathbf{U}) \mathbf{U}
$$

and

$$
\mathbf{G}=\frac{\partial \mathbf{G}}{\partial \mathbf{U}} \mathbf{U}=\mathbf{B}(\mathbf{U}) \mathbf{U}
$$

where $\mathbf{A}$ and $\mathbf{B}$ are given in app. I.

We assume that these equations are defined on a physical space $\Phi$. In this space is included a subdomain $\Omega$ whose boundary is denoted by $\Gamma$. On the boundary, we define a curvilinear coordinate $s$ and a normal $\mathbf{n}=\left(n_{x}, n_{y}\right)$ that points outward. (See fig. 1.)

The optimization problem studied here is defined as the minimization of the functional $\mathcal{E}=\int_{\Gamma} \phi(p, \rho, u, v, \Gamma) d s$ over all admissible shapes of the subdomain $\Omega$, subject to the steady-state Euler equations with proper boundary conditions on $\Gamma$.

Although the method we present is general, we focus on the following model problem. The subdomain $\Omega$ is represented by a nozzle. (See fig. 2). At the inlet, total pressure, total temperature, and the ratio $\sigma=v / u$ are fixed. At the outlet, if the flow is subsonic, the static pressure is fixed, and at the solid walls the impermeability condition $u n_{x}+v n_{y}=$ 0 is enforced. The upper wall is kept fixed. The lower wall $\Theta$ is represented by the parameterization

$$
y(\Theta)=\sum_{i} \alpha_{i} f_{i}(x)
$$

where the functions $f_{i}(x)$ are some shape functions and $\alpha=\left(\alpha_{1}, \ldots, \alpha_{i}, \ldots\right)$ is the corresponding set of shape coefficients. Given a desirable lower wall pressure distribution $p^{*}(x)$ and the actual pressure distribution on the lower wall $p^{w}(x)$, the optimization problem consists in finding a set of shape coefficients $\alpha_{i}$ such that the functional

$$
\mathcal{E}=\frac{1}{2} \int_{a}^{b}\left(p^{w}-p^{*}\right)^{2} d x
$$

is minimized. 


\section{$3 \quad$ Lagrange Multipliers and Optimality}

The problem of achieving the minimum is addressed by introducing a set of Lagrange multipliers. Consider the augmented functional

$$
\mathcal{L}(\mathbf{U}, \alpha, \boldsymbol{\Lambda}, \mu)=\mathcal{E}+\int_{\Omega}{ }^{t} \boldsymbol{\Lambda}\left(\mathbf{A} \mathbf{U}_{x}+\mathbf{B U}_{y}\right) d \Omega+\int_{\Theta} \mu \rho \mathbf{V} \cdot \mathbf{n} d s
$$

where $\mathbf{V}=(u, v)$. The vector $\mathbf{\Lambda}(x, y)={ }^{t}\left(\lambda_{1}, \lambda_{2}, \lambda_{3}, \lambda_{4}\right)$ and the scalar $\mu(s)$ are the continuous equivalents of the Lagrange multipliers.

We calculate the variation of the functional $\mathcal{L}$ with respect to the variation of the functions $\mathbf{U}, \boldsymbol{\Lambda}$, and $\mu$ and the parameters $\alpha_{i}$, respectively. When $\mathbf{U}(x, y)$ is increased by a function $\varepsilon \widetilde{\mathbf{U}}(x, y)$, the functional $\mathcal{L}$ increases by an amount $\varepsilon \delta \mathcal{L}_{U}$. In the same way, $\boldsymbol{\Lambda}(x, y)$ is increased by $\varepsilon \tilde{\boldsymbol{\Lambda}}(x, y) ; \mu(s)$, by $\varepsilon \tilde{\mu}(s)$; and each $\alpha_{i}$, by $\varepsilon \tilde{\alpha}_{i}$.

If we follow the derivation in app. II and take

$$
\delta \mathcal{L}=\delta \mathcal{L}_{U}+\delta \mathcal{L}_{\Lambda}+\delta \mathcal{L}_{\mu}+\delta \mathcal{L}_{\alpha}
$$

then we obtain

$$
\begin{aligned}
\delta \mathcal{L}_{U} & =\left.\int_{a}^{b} \frac{\partial p}{\partial \mathbf{U}}\right|_{\Theta}\left(p^{w}-p^{*}\right) \widetilde{\mathbf{U}} d x+\int_{\Gamma}{ }^{t} \boldsymbol{\Lambda}\left(\mathbf{A} n_{x}+\mathbf{B} n_{y}\right) \widetilde{\mathbf{U}} d s+ \\
& -\int_{\Omega}\left({ }^{t} \boldsymbol{\Lambda}_{x} \mathbf{A}+{ }^{t} \boldsymbol{\Lambda}_{y} \mathbf{B}\right) \widetilde{\mathbf{U}} d \Omega+\int_{\Theta} \mu \mathbf{n} \frac{\partial \rho \mathbf{V}}{\partial \mathbf{U}} \widetilde{\mathbf{U}} d s
\end{aligned}
$$

where

$$
\frac{\partial p}{\partial \mathbf{U}}=2 k\left(\frac{u^{2}+v^{2}}{2},-u,-v, 1\right) \text { and } \frac{\partial \rho \mathbf{V}}{\partial \mathbf{U}}=\left(\begin{array}{cccc}
0 & 1 & 0 & 0 \\
0 & 0 & 1 & 0
\end{array}\right)
$$

Furthermore,

$$
\begin{aligned}
\delta \mathcal{L}_{\Lambda} & =\int_{\Omega}{ }^{t} \tilde{\mathbf{\Lambda}}\left(\mathbf{A} \mathbf{U}_{x}+\mathbf{B} \mathbf{U}_{y}\right) d \Omega \\
\delta \mathcal{L}_{\mu} & =\int_{\Theta} \tilde{\mu} \rho \mathbf{V} \cdot \mathbf{n} d s \\
\delta \mathcal{L}_{\alpha} & =\sum_{i}\left[\left.\int_{a}^{b} \frac{d p}{d y}\right|_{\Theta}\left(p^{w}-p^{*}\right) f_{i} d x+\int_{\Theta}{ }^{t} \boldsymbol{\Lambda}\left(\mathbf{A} \mathbf{U}_{x}+\mathbf{B} \mathbf{U}_{y}\right) f_{i} \cos \theta d s\right. \\
& +\int_{\Theta} \mu \frac{\partial(\rho \mathbf{V})}{\partial y} \cdot \mathbf{n} f_{i} d s-\int_{\Theta} \mu \rho \mathbf{V} \cdot \mathbf{t} \frac{d f_{i}}{d x} \cos ^{2} \theta d s \\
& \left.+\int_{\Theta} \mu \rho \mathbf{V} \cdot \mathbf{n} \frac{d f_{i}}{d x} \sin \theta \cos \theta d s\right] \tilde{\alpha}_{i}
\end{aligned}
$$

where $\theta$ is the angle between the normal $\mathbf{n}$ and the $y$-axis and $\mathbf{t}=\left(-n_{y}, n_{x}\right)$. 
At the minimum of the functional, we have $\delta \mathcal{L}=0$ for all possible choices of the functions $\widetilde{\mathbf{U}}, \tilde{\boldsymbol{\Lambda}}$, and $\tilde{\mu}$ and of the parameters $\tilde{\alpha}$. This condition is reached when

$$
\delta \mathcal{L}_{U}=\delta \mathcal{L}_{\Lambda}=\delta \mathcal{L}_{\mu}=\delta \mathcal{L}_{\alpha}=0
$$

Note that because of the necessary conditions (eqs. (11)) the unconstrained minimum of the functional $\mathcal{L}(\mathrm{U}, \alpha, \boldsymbol{\Lambda}, \mu)$ corresponds to the constrained minimum of the functional $\mathcal{E}(\alpha)$. In fact, we have

$$
\delta \mathcal{L}_{\Lambda}=0 \Rightarrow \mathbf{A} \mathbf{U}_{x}+\mathbf{B} \mathbf{U}_{y}=\mathbf{0} \text { and } \delta \mathcal{L}_{\mu}=0 \Rightarrow \rho \mathbf{V} \cdot \mathbf{n}=0
$$

which means that $\mathbf{U}$ must satisfy the Euler equations with boundary conditions. In addition, for the minimum of $\mathcal{L}$ we have $\delta \mathcal{L}_{U}=0$, which leads to

$$
\begin{gathered}
{ }^{t} \mathbf{A} \boldsymbol{\Lambda}_{x}+{ }^{t} \mathbf{B} \boldsymbol{\Lambda}_{y}=\mathbf{0} \text { on } \Omega \\
\left.\frac{\partial p}{\partial \mathbf{U}}\right|_{\Theta}\left(p^{w}-p^{*}\right) \cos \theta+{ }^{t} \boldsymbol{\Lambda}\left(\mathbf{A} n_{x}+\mathbf{B} n_{y}\right)+\mu \mathbf{n} \frac{\partial \rho \mathbf{V}}{\partial \mathbf{U}}=\mathbf{0} \text { on } \Theta
\end{gathered}
$$

At the inlet, outlet, and upper wall,

$$
{ }^{t} \boldsymbol{\Lambda}\left(\mathbf{A} n_{x}+\mathbf{B} n_{y}\right) \widetilde{\mathbf{U}}=0
$$

Given $\mathbf{U}$ and the set of costate eqs. (12) and (14), we can determine uniquely $\boldsymbol{\Lambda}$ in $\Omega$ and $\mu$ on $\Theta$. (See app. III).

Finally, given $\alpha$ and given $\mathbf{U}$ and $\boldsymbol{\Lambda}$ from the above equations, we can calculate from eq. (10)

$$
\begin{aligned}
\frac{\partial \mathcal{L}}{\partial \alpha_{i}} & =\left.\int_{a}^{b} \frac{d p}{d y}\right|_{\Theta}\left(p^{w}-p^{*}\right) f_{i} d x+\int_{\Theta}{ }^{t} \boldsymbol{\Lambda}\left(\mathbf{A} \mathbf{U}_{x}+\mathbf{B} \mathbf{U}_{y}\right) f_{i} \cos \theta d s \\
& +\int_{\Theta} \mu \frac{\partial(\rho \mathbf{V})}{\partial y} \cdot \mathbf{n} f_{i} d s-\int_{\Theta} \mu \rho \mathbf{V} \cdot \mathbf{t} \frac{d f_{i}}{d x} \cos ^{2} \theta d s \\
& +\int_{\Theta} \mu \rho \mathbf{V} \cdot \mathbf{n} \frac{d f_{i}}{d x} \sin \theta \cos \theta d s
\end{aligned}
$$

In cases for which shock occurs in the flow field, we split the domain of integration by means of a curve $\Upsilon$ that coincides with the shock where it exists. Then, we follow the same derivation so far on each of the two subdomains, with $\Upsilon$ as a boundary. (See app. III.) The strategy that we use to achieve the minimum of $\mathcal{L}$ is as follows:

1. Start with a set $\alpha$ of shape coefficients.

2. Enforce $\delta \mathcal{L}_{\Lambda}=0$ and $\delta \mathcal{L}_{\mu}=0$ by finding $\mathbf{U}$ such that it satisfies the steady-state Euler equations and boundary conditions.

3. Enforce $\delta \mathcal{L}_{U}=0$ by finding $\Lambda$ such that it satisfies the costate equations and boundary conditions. 
4. Calculate $\nabla_{\alpha} \mathcal{L}$. If $\nabla_{\alpha} \mathcal{L}=0$ then we have determined the minimum; otherwise continue to steps 5 and 6 .

5. Update $\alpha$ with criteria based on $\nabla_{\alpha} \mathcal{L}$.

6. Restart from step 2.

\section{Discrete Problem}

We introduce a discrete grid that is defined as $\left(x_{l}, y_{m}\right)=\left(x_{0}+l \Delta x, y(\Theta)+m \Delta y\right)$, where $\Delta x$ is constant and $\Delta y$ is a constant fraction of the local height of the nozzle. (See fig. 3.) The steady solution of the Euler equations is obtained with a time-dependent technique, in the frame of an explicit finite-volume code. The conservative variables $\mathbf{U}$ are computed at the cell centers, and the fluxes $\mathbf{F}$ and $\mathbf{G}$ are evaluated at the cell interfaces with the approximate Riemann solver in reference [7]. Second-order accuracy is achieved by using an essentially nonoscillatory scheme [2]. The flow-field values at the cell interfaces, used as initial conditions for the Riemann problem, are reconstructed by means of a linear interpolation and a minmod limiter. The amplitude of the integration step is chosen in accordance with the Courant-Friedrichs-Lewy (CFL) condition.

The costate equations are discretized on the same grid presented above. Because they have no conservative form, the numerical solution is obtained with a finite-difference scheme. We introduce a set of curvilinear coordinates $\varphi(x, y)$ and $\psi(x, y)$. The costate equations are then written

$$
{ }^{t} \mathcal{A} \boldsymbol{\Lambda}_{\varphi}+{ }^{t} \mathcal{B} \boldsymbol{\Lambda}_{\psi}=\mathbf{0}
$$

where $\mathcal{A}=\mathbf{A} \varphi_{x}+\mathbf{B} \varphi_{y}$ and $\mathcal{B}=\mathbf{A} \psi_{x}+\mathbf{B} \psi_{y}$. The transformations $\varphi$ and $\psi$ are defined as $\left(x_{l}, y_{m}\right) \stackrel{\varphi}{\rightarrow} l$ and $\left(x_{l}, y_{m}\right) \stackrel{\psi}{\rightarrow} m$, respectively.

We find the solution of eq.(16) as the asymptotic limit of a time-dependent technique. Consider eq. (16) embedded in time as

$$
\pm \boldsymbol{\Lambda}_{t}+{ }^{t} \mathcal{A} \boldsymbol{\Lambda}_{\varphi}+{ }^{t} \mathcal{B} \boldsymbol{\Lambda}_{\psi}=\mathbf{0}
$$

We must select the proper sign for the time derivative. The inlet and outlet boundary conditions for the costate equations are complementary to those of the flow field equations, in the sense that if the number of boundary conditions for the flow field is $c$, then the number of boundary conditions for the costate equations is $4-c$. Therefore, the above equations and boundary conditions are well posed if we select the negative sign for the time derivative. In fact, the resulting characteristic pattern is mirror symmetric with respect to that of the flow-field equations.

In the presence of a shock in the flow field, the matrices ${ }^{t} \mathcal{A}$ and ${ }^{t} \mathcal{B}$ are discontinuous. In particular, the characteristic pattern at the shock indicates the necessity of a boundary condition for the costate equations. For further discussion, see reference [3].

The costate equations are linear and as such are the boundary conditions. We exploit this property to numerically solve these equations. Suppose that locally we separate the 
variables through the following approximation:

$$
\boldsymbol{\Lambda}(\varphi, \psi, t)=\mathbf{\Lambda}^{\prime}(\varphi, t)+\mathbf{\Lambda}^{\prime \prime}(\psi, t) .
$$

This separation of variables means that, for example, in a Taylor expansion about the point $(\varphi, \psi)$ we disregard all terms that involve a cross product $\varphi \psi$. This approximation is at least first-order accurate. We substitute eq. (18) into eq. (17) to obtain

$$
-\boldsymbol{\Lambda}_{t}^{\prime}-\boldsymbol{\Lambda}^{\prime \prime}{ }_{t}+{ }^{t} \mathcal{A} \boldsymbol{\Lambda}_{\varphi}^{\prime}+{ }^{t} \mathcal{B} \boldsymbol{\Lambda}_{\psi}^{\prime \prime}=\mathbf{0}
$$

and we are left with the following subproblems in one dimension:

$$
\begin{aligned}
& -\Lambda_{t}^{\prime}+{ }^{t} \mathcal{A} \Lambda_{\varphi}^{\prime}=0 \\
& -\Lambda^{\prime \prime}{ }_{t}+{ }^{t} \mathcal{B} \Lambda_{\psi}^{\prime \prime}=0
\end{aligned}
$$

Let us define $\mathbf{n}_{\varphi}=\left(\varphi_{x} / \sqrt{\varphi_{x}^{2}+\varphi_{y}^{2}}, \varphi_{y} / \sqrt{\varphi_{x}^{2}+\varphi_{y}^{2}}\right)$ and $\mathbf{n}_{\psi}=\left(\psi_{x} / \sqrt{\psi_{x}^{2}+\psi_{y}^{2}}, \psi_{y} / \sqrt{\psi_{x}^{2}+\psi_{y}^{2}}\right)$. The left and right eigenvector matrices of $\mathcal{A}$ and $\mathcal{B}$ are calculated by using the formulas in App. I with $\mathbf{n}=\mathbf{n}_{\varphi}$ and $\mathbf{n}=\mathbf{n}_{\psi}$, respectively. After eqs. (19) and (20) are diagonalized, we upwind the derivatives of the characteristic variables according to the signs of the corresponding eigenvalues. The time step $\Delta t$ is chosen according to the CFL condition.

This method can be regarded as a two-dimensional interpretation of the method presented in reference [6].

The boundary conditions can be split in a similar way. Consider, for example, the boundary condition at the solid wall. Because eq. (19) is defined along the wall, the characteristic variables can be upwinded according to the corresponding eigenvalues. On the contrary, the third row of eq. (20), which corresponds to the characteristic with a speed of $+a$, is replaced by the boundary condition in eq. (AIII.5). Note that the contravariant component of the speed in the direction $\psi$ is 0 ; therefore, the resulting system can be written as

$$
\left\{\begin{aligned}
\Delta^{t} W_{1}^{\prime \prime} & =0 \\
\Delta^{t} W_{2}^{\prime \prime} & =0 \\
n_{x} \Delta^{t} \lambda_{2}^{\prime \prime}+n_{y} \Delta^{t} \lambda_{3}^{\prime \prime} & =-\left(p^{w}-p^{*}\right) \cos \theta-n_{x} \Delta^{t} \lambda_{2}^{\prime}-n_{y} \Delta^{t} \lambda_{3}^{\prime} \\
\Delta^{t} W_{4}^{\prime \prime} & =a \Delta t\left(\sqrt{\psi_{x}^{2}+\psi_{y}^{2}}\right) \Delta^{m} W_{4}^{\prime \prime}
\end{aligned}\right.
$$

where $\Delta(\cdot)$ is the forward finite increment of the function $(\cdot)$ with respect to the superscripted variable and

$$
\Delta \mathbf{W}=\left(\begin{array}{c}
\Delta W_{1} \\
\Delta W_{2} \\
\Delta W_{3} \\
\Delta W_{4}
\end{array}\right)={ }^{t} \mathbf{L}_{n}^{-1} \Delta \mathbf{\Lambda}
$$

In the third row of eq. (21), we have the functions of $\Delta^{t} \boldsymbol{\Lambda}^{\prime}$, which are computed separately as mentioned. The other boundary conditions are enforced in the same pattern that is presented above. 


\section{Optimization Experiments}

The optimization problem is addressed with four different gradient-based criteria.

1. Steepest descent (SD1). The shape coefficients are updated as follows: $\alpha_{i} \leftarrow \alpha_{i}-v\left(\partial \mathcal{L} / \partial \alpha_{i}\right)$, where $v$ is a given parameter.

2. Steepest descent with $v$ selected as shown (SD2). Because we know the gradient $\nabla_{\alpha} \mathcal{L}$ at the present iteration, we can use a tentative $v$ and can compute the gradient $\nabla_{\alpha} \mathcal{L}^{\prime}$. By calculating $\nabla_{\alpha} \mathcal{L} \cdot \nabla_{\alpha} \mathcal{L}^{\prime}$, we linearly estimate $v$ such that eventually $\nabla_{\alpha} \mathcal{L} \cdot \nabla_{\alpha} \mathcal{L}^{\prime \prime}=0$. Each step of the optimization requires solution of the flow-field and costate equations twice.

3. The BFGS algorithm (presented in reference [9]). This algorithm (BFGS1) accounts for the curvature of the hypersurface $\mathcal{L}$. The shape coefficients are updated according to the formula $\alpha_{i} \leftarrow \alpha_{i}-v d_{i}$, where $\mathbf{d}=\left(\ldots, d_{i}, \ldots\right)$ is the descent direction determined by $\mathbf{d}=\mathbf{H} \nabla_{\alpha} \mathcal{L}$ and $\mathbf{H}$ is an estimate of the inverse of the Hessian of the functional.

4. The BFGS algorithm (as above) with a linear estimate of $v$ such that $\mathbf{d} \cdot \nabla_{\alpha} \mathcal{L}^{\prime \prime}=$ 0 . Each optimization step requires solution of the flow-field and costate equations twice (BFGS2).

The computations are performed on a $40 \times 20$ grid unless otherwise specified. Total pressure and total temperature at the inlet are taken unitary and $\sigma(0, y)=0$. At the outlet, the static pressure depends on the test case considered. For the lower wall ordinate $y(\Theta)$, we have

$$
y(\Theta)= \begin{cases}0 & \text { if }-0.5 \leq x<0 \\ \sum_{i=1}^{4} \alpha_{i} x^{i+1}(x-1)^{2} & \text { if } 0 \leq x<1 \\ 0 & \text { if } 1 \leq x<1.5\end{cases}
$$

The optimization consists in finding the four shape coefficients $\alpha_{i}$ such that the modulus of the gradient $\nabla_{\alpha} \mathcal{L}$ is 0 . The flow-field and costate equations are iterated until the residuals are less than $10^{-5}$.

Consider a test case in which a pressure distribution in a subsonic nozzle is found for which the outlet pressure is 0.9 of the inlet total pressure. We take $\alpha=(2,0,0,0)$ and define the corresponding configuration as the optimal configuration. Then, we compute the flow field and determine the pressure distribution on the lower wall. This pressure distribution $p^{*}$ is the one we want to recover with the optimization algorithm. In fig. 6 , the target flow field and the starting configuration, obtained with $\alpha=(-2,0,0,0)$, are shown along with the convergence histories for DS1 and BFGS2.

For the supersonic case, we take a constant section channel as a starting configuration and $\alpha=(2,0,0,0)$ as the target. In fig. 7 , we present the results obtained when the outlet pressure is lowered to 0.5 of the inlet total pressure. A relevant shock is generated in the flow field. In the first optimization iterations, we updated the shape coefficients, as was done for DS1. This step is necessary because this far from the minimum the functional 
$\mathcal{L}$ might be not convex; therefore, the estimate of $v$ that was used would not be correct. Figure 8 shows the sequence of lower wall configurations obtained with BFGS2. We also tested the effect of grid coarseness by reducing the number of grid points to $20 \times 10$. (See fig. 9.)

The second test case is designed to check the capability of the algorithm in detecting minima in cases for which the desired pressure distribution is out of the design space (i.e., the functional is not 0 at the minimum). The pressure distribution $p^{*}$ is obtained with an outlet boundary condition that differs from the distribution that is actually used in the optimization routine. The results are given in fig. 10 .

The DS1 updating strategy had the least attractive rate of reduction of the functional. Our experience showed, nevertheless, that it was the most reliable in cases of complicated surface topologies that can occur in flow fields with embedded shocks. The BFGS2 becomes the most efficient of the algorithms tested when it is coupled with DS1. With this algorithm, the functional was reduced by orders of magnitude.

Each optimization procedure with a $40 \times 20$ grid required $6 \mathrm{~h}$ of central processing unit (cpu) time on a DEC 3000/500. The $20 \times 10$ case required $1 \mathrm{~h}$ of $\mathrm{cpu}$ time.

\section{Concluding Remarks}

We have derived an expression of the gradient of the cost function with respect to the shape coefficients. The boundary conditions for the costate equations have been presented; we have shown their relevance to the well posedness of the problem. In the case of shocks, we provided the proper conditions for the costate equations at the discontinuity. On the discrete level, we proposed a method of integrating the costate equations in accordance with a revisited scheme. Additional work is needed to test the algorithm with more realistic test cases and to apply the One-Shot method (reference [10]) to hyperbolic problems.

\section{Acknowledgments}

Many discussions with S. Ta'asan and M. Pandolfi contributed to the formulation of the problem and to its numerical solution.

\section{References}

[1] Ed. Miele A. Theory of Optimum Aerodynamic Shapes. Academic Press, New York, 1965.

[2] Harten A., Engquist B., and Chakravarthy S.R. Uniformly high order accurate essentially non-oscillatory schemes, iii. Journal of Computational Physics, vol. 71, 1987.

[3] Iollo A., Salas M.D., and Ta'asan S. Shape optimization governed by the euler equations using an adjoint method. Technical Report 93-78, ICASE, 1993. 
[4] Jameson A. Aerodynamic design via control theory. Technical Report 88-64, ICASE, 1988.

[5] Beaux F. and Dervieux A. Exact-gradient shape optimization of a $2 \mathrm{~d}$ euler flow. Finite Elements in Analysis and Design, vol. 12:281-302, 1992.

[6] Moretti G. The $\lambda$ scheme. Comput. Fluids, vol. 7:191-205, 1979.

[7] Pandolfi M. A contribution to the numerical prediction of unsteady flows. AIAA J., vol. $22(5), 1984$.

[8] Lighthill M.J. A new method of two dimensional aerodynamic design. ARC Rand $M$ 2112, 1945 .

[9] Fletcher R. Practical Methods of Optimization, volume I. John Wiley \& Sons, 1980.

[10] Ta'asan S., Kuruvila G., and Salas M.D. Aerodynamic design and optimization in one shot. In 30th Aerospace Sciences Meeting and Exhibit, AIAA 92-005, Jan. 1992. 


\section{Appendix I}

The Jacobian matrices for the Euler equation in conservative variables are

$$
\begin{aligned}
\mathbf{A} & =\left[\begin{array}{cccc}
0 & 1 & 0 & 0 \\
k V^{2}-u^{2} & (3-\gamma) u & -2 k v & 2 k \\
-u v & v & u & 0 \\
\left(-\gamma e+2 k V^{2}\right) u & \gamma e-k V^{2}-2 k u^{2} & -2 k u v & \gamma u
\end{array}\right] \\
\mathbf{B} & =\left[\begin{array}{cccc}
0 & 0 & 1 & 0 \\
-u v & v & u & 0 \\
k V^{2}-v^{2} & -2 k u & (3-\gamma) v & 2 k \\
\left(-\gamma e+2 k V^{2}\right) v & -2 k u v & \gamma e-k V^{2}-2 k v^{2} & \gamma v
\end{array}\right]
\end{aligned}
$$

The Jacobian in the direction $\mathbf{n}$ is $\mathbf{C}=\mathbf{A} n_{x}+\mathbf{B} n_{y}$. The left $\left(\mathbf{L}_{n}\right)$ and right $\left(\mathbf{L}_{n}^{-1}\right)$ eigenvector matrices of $\mathbf{C}$ are

$$
\begin{aligned}
\mathbf{L}_{n} & =\left[\begin{array}{cccc}
1-k V^{2} / a^{2} & 2 k u / a^{2} & 2 k v / a^{2} & -2 k / a^{2} \\
V_{t} / \rho & n_{y} / \rho & -n_{x} / \rho & 0 \\
\left(-V_{n}+k V^{2} / a\right) / \rho & \left(n_{x}-2 k u / a\right) / \rho & \left(n_{y}-2 k v / a\right) / \rho & 2 k / \rho a \\
\left(V_{n}+k V^{2} / a\right) / \rho & -\left(n_{x}+2 k u / a\right) / \rho & -\left(n_{y}+2 k v / a\right) / \rho & 2 k / \rho a
\end{array}\right] \\
\mathbf{L}_{n}^{-1} & =\left[\begin{array}{cccc}
1 & 0 & \rho / 2 a & \rho / 2 a \\
u & \rho n_{y} & \rho\left(u+a n_{x}\right) / 2 a & \rho\left(u-a n_{x}\right) / 2 a \\
v & \rho n_{x} & \rho\left(v+a n_{y}\right) / 2 a & \rho\left(v-a n_{y}\right) / 2 a \\
V^{2} / 2 & -\rho V_{t} & \rho\left(V^{2}+a^{2} / k+2 a V_{n}\right) / 4 a & \rho\left(V^{2}+a^{2} / k-2 a V_{n}\right) / 4 a
\end{array}\right]
\end{aligned}
$$

where $V_{n}=\mathbf{V} \cdot \mathbf{n}$ and $V_{t}=\mathbf{V} \cdot \mathbf{t}$. The diagonal matrix $\mathbf{D}_{n}=\mathbf{L}_{n} \mathbf{C}_{n} \mathbf{L}_{n}^{-1}$ is

$$
\mathbf{D}_{n}=\left[\begin{array}{cccc}
V_{n} & 0 & 0 & 0 \\
0 & V_{n} & 0 & 0 \\
0 & 0 & V_{n}+a & 0 \\
0 & 0 & 0 & V_{n}-a
\end{array}\right]
$$

\section{Appendix II}

To calculate $\delta \mathcal{L}_{U}$, consider the increment $\mathbf{U} \leftarrow \mathbf{U}+\varepsilon \widetilde{\mathbf{U}} \Rightarrow$

$$
\mathbf{F} \leftarrow \mathbf{F}+\varepsilon \mathbf{A} \widetilde{\mathbf{U}}+\text { h.o.t. and } \mathbf{G} \leftarrow \mathbf{G}+\varepsilon \mathbf{B} \widetilde{\mathbf{U}}+\text { h.o.t. }
$$

We obtain

$$
\begin{aligned}
\delta \mathcal{L}_{U} & \left.=\left.\int_{a}^{b} \frac{\partial p}{\partial \mathbf{U}}\right|_{\Theta}\left(p-p^{*}\right) \widetilde{\mathbf{U}} d x+\int_{\Omega}{ }^{t} \boldsymbol{\Lambda}\left[(\mathbf{A} \widetilde{\mathbf{U}})_{x}+\mathbf{B} \widetilde{\mathbf{U}}\right)_{y}\right] d \Omega \\
& +\int_{\Theta} \mu \mathbf{n} \frac{\partial \rho \mathbf{V}}{\partial \mathbf{U}} \widetilde{\mathbf{U}} d s+\text { h.o.t. }
\end{aligned}
$$


If we apply Gauss' theorem to the second integral of the above equation then we find eq. (7). Equations (8) and (9) are easily obtained.

To calculate $\delta \mathcal{L}_{\alpha}$, we first consider the variation of the functions defined on $\Theta$ :

$$
\alpha_{i} \leftarrow \alpha_{i}+\varepsilon \tilde{\alpha}_{i} \Rightarrow p^{w} \leftarrow p^{w}+\varepsilon \frac{d p}{d y} f_{i} \tilde{\alpha}_{i} \text { and } \rho \mathbf{V} \leftarrow \rho \mathbf{V}+\varepsilon \frac{\partial \rho \mathbf{V}}{\partial y} f_{i} \tilde{\alpha}_{i}
$$

Then, we consider the variation of the geometry; other higher order effects are disregarded. When the geometry is perturbed, the domain of integration $\Omega$, the normal $\mathbf{n}$ and the element of integration $d s$ are perturbed. The domain $\Omega$ is increased (fig. 4) by a quantity $\varepsilon \tilde{\alpha}_{i} f_{i} \cos \theta d s$. The normal $\mathbf{n}$ is perturbed by a quantity $-\varepsilon \tilde{\alpha}_{i} d f_{i} / d x \cos ^{2} \theta \mathbf{t} ; d s$, by $\varepsilon \tilde{\alpha}_{i} d f_{i} / d x \cos \theta \sin \theta d s$. (See fig. 5.)

\section{Appendix III}

Consider eq. (14). This equation defines the boundary conditions for $\boldsymbol{\Lambda}$ after we impose the proper constraints on $\widetilde{\mathbf{U}}$. At the inlet, only one component of the variation of the flux in the direction normal to the boundary $\widetilde{\mathbf{F}}_{n}=\left(\mathbf{A} n_{x}+\mathbf{B} n_{y}\right) \widetilde{\mathbf{U}}$ is independent of the others because total pressure, total temperature, and $\sigma$ are fixed. If we express all components of $\widetilde{\mathbf{F}}_{n}$ in terms of $\widehat{\rho u}$, we obtain

$$
\widetilde{\mathbf{F}}_{n}=\left\{\begin{array}{c}
\xi \\
{\left[\xi+\eta \sigma /\left(1-M^{2}\right)\right] u} \\
{\left[\sigma \xi-\eta /\left(1-M^{2}\right)\right] u} \\
H \xi
\end{array}\right\} \widetilde{\rho u}
$$

where $\xi=n_{x}+\sigma n_{y}, \eta=\left(n_{y}-\sigma n_{x}\right), H$ is the specific total enthalpy, and $M$ is the local Mach number. For an arbitrary choice of $\widetilde{\rho u}$, from eq. (14) we have

$$
\lambda_{1} \xi+\lambda_{2} u\left[\xi+\eta \sigma /\left(1-M^{2}\right)\right]+\lambda_{3} u\left[\sigma \xi-\eta /\left(1-M^{2}\right)\right]+\lambda_{4} H \xi=0
$$

At the outlet, if the flow is subsonic, then only the static pressure is fixed; therefore, three components of the vector $\widetilde{\mathbf{U}}$ are arbitrary. If we take $\widetilde{\rho e}$ as the dependent variable, we have

$$
\widetilde{\mathbf{F}}_{n}=\left[\begin{array}{c}
\widetilde{\rho u} n_{x}+\widetilde{\rho v} n_{y} \\
\widetilde{\rho u}\left(V_{n}+u n_{x}\right)+u n_{y} \widetilde{\rho v}-\tilde{\rho} u V_{n} \\
\widetilde{\rho v}\left(V_{n}+v n_{y}\right)+v n_{x} \widetilde{\rho u}-\tilde{\rho} v V_{n} \\
-\tilde{\rho}\left(\gamma p / 2 k \rho+u^{2}+v^{2}\right)+\widetilde{\rho} u\left(V_{n} u+H n_{x}\right)+\widetilde{\rho v}\left(V_{n} v+H n_{y}\right)
\end{array}\right]
$$

For an arbitrary choice of $\tilde{\rho}, \widetilde{\rho u}$, and $\widetilde{\rho v}$, from eq. (14) we have

$$
\begin{array}{r}
\lambda_{1} n_{x}+\lambda_{2}\left(V_{n}+u n_{x}\right)+\lambda_{3} v n_{x}+\lambda_{4}\left(V_{n} u+H n_{x}\right)=0 \\
\lambda_{1} n_{y}+\lambda_{2} u n_{y}+\lambda_{3}\left(V_{n}+v n_{y}\right)+\lambda_{4}\left(V_{n} v+H n_{y}\right)=0 \\
\lambda_{2} u V_{n}+\lambda_{3} v V_{n}+\lambda_{4} V_{n}\left(\gamma p / 2 k \rho+u^{2}+v^{2}\right)=0
\end{array}
$$


For a supersonic outlet, no conditions exist on $\widetilde{\mathbf{U}}$; therefore, we have

$$
\boldsymbol{\Lambda}=\mathbf{0} \text {. }
$$

If a shock is embedded in the flow field, then the shock is considered as a boundary for the costate equations. The consequent boundary conditions are applied on each side of eq. (AIII.3) before the shock and eq. on each side of (AIII.1) after the shock.

For the upper wall, we have

$$
\tilde{\mathbf{F}}_{n}=\left[\begin{array}{c}
0 \\
n_{x} \\
n_{y} \\
0
\end{array}\right] \tilde{p}
$$

such that for an arbitrary choice of $\tilde{p}$ eq. (14) is satisfied if

$$
\lambda_{2} n_{x}+\lambda_{3} n_{y}=0
$$

At the lower wall, eq. (13) applies. Because the rank of $\mathbf{A} n_{x}+\mathbf{B} n_{y}$ at the wall is 2, the system has only two linearly independent rows. We obtain

$$
\lambda_{2} n_{x}+\lambda_{3} n_{y}+\left(p^{w}-p^{*}\right) \cos \theta=0
$$

which is the boundary condition for $\boldsymbol{\Lambda}$, and

$$
\mu=-\left[\lambda_{1}+u \lambda_{2}+v \lambda_{3}+\left(\gamma e-k V^{2}\right) \lambda_{4}\right]
$$

which is the relation between $\mu$ and $\boldsymbol{\Lambda}$ on the boundary. 


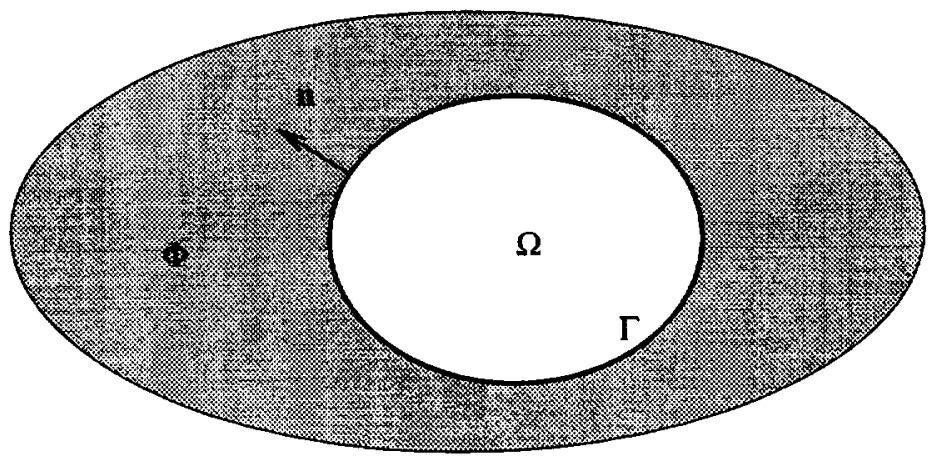

Figure 1: Physical space.

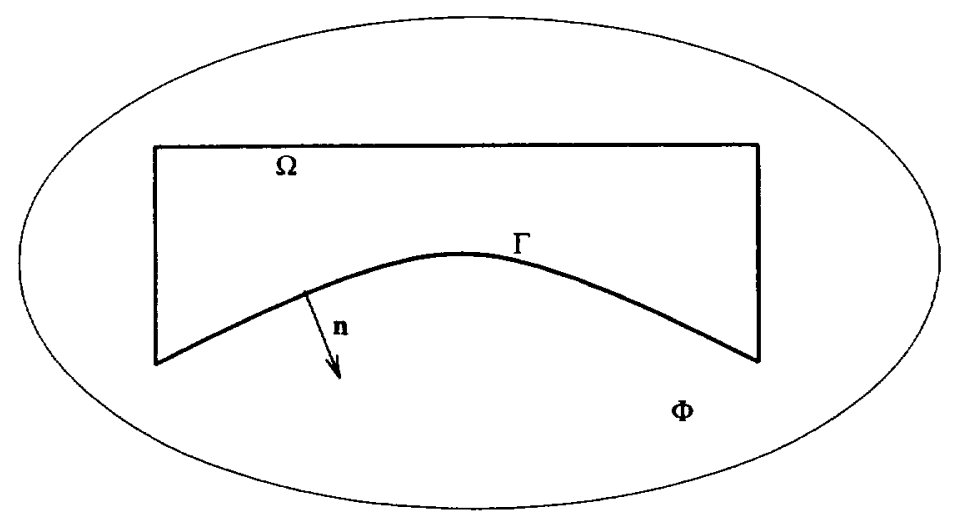

Figure 2: Model problem. 


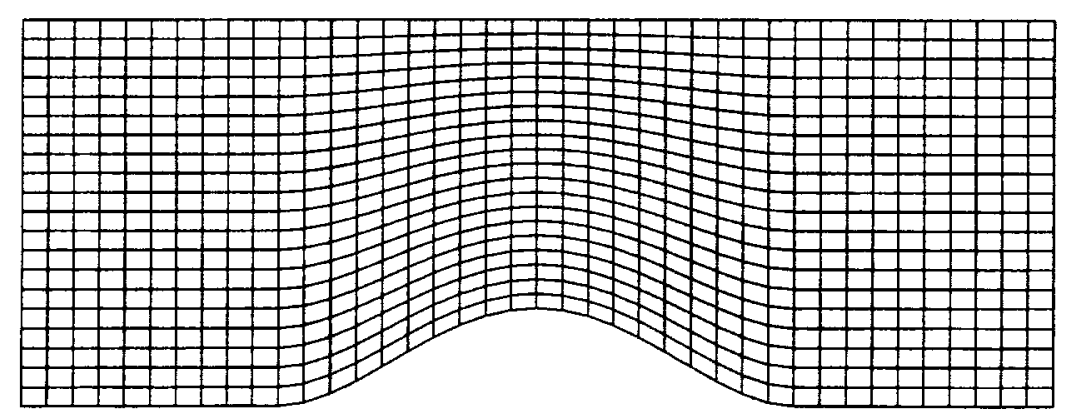

Figure 3: Discrete grid.

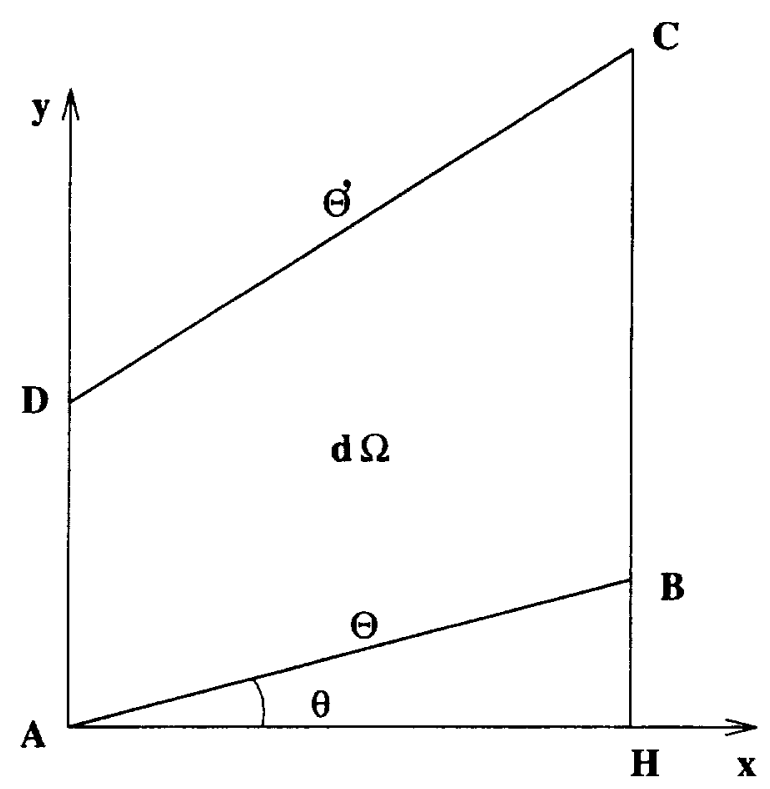

Figure 4: Variation of domain of integration $\Omega$ with $A H=d x, A D=\varepsilon \tilde{y}$ and $B C=$ $\varepsilon(\tilde{y}+\partial \tilde{y} / \partial x d x)$. 


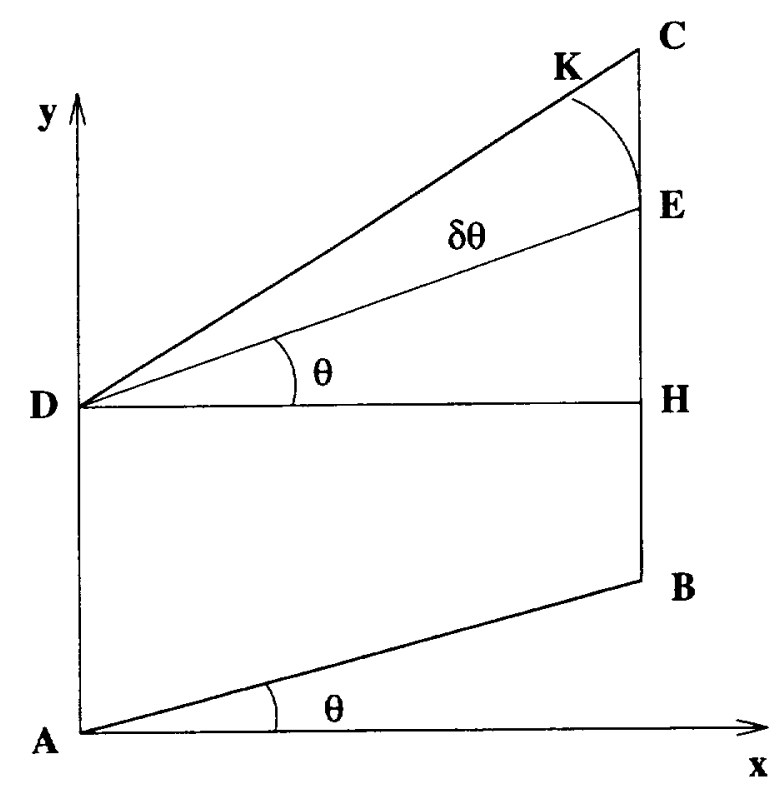

Figure 5: Variation of $\mathbf{n}$ and $d s$ with $C E=\varepsilon \partial \tilde{y} / \partial x d x, D H=d x, D E=d s, \varepsilon \delta \theta=E K / d s$, and $E K=C E \cos \theta$ (i.e., $\delta \theta=\partial \tilde{y} / \partial x \cos ^{2} \theta$ ); variation of $d s$ is $K C=\varepsilon \partial \tilde{y} / \partial x \sin \theta d x$ 


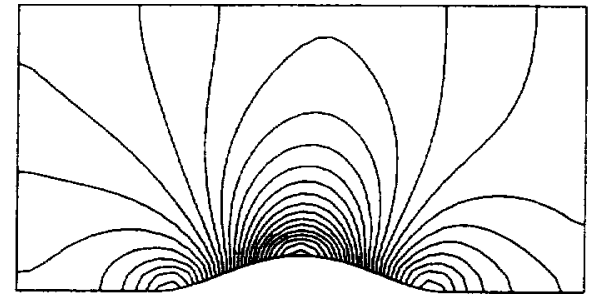

(a) Target Mach-number field.

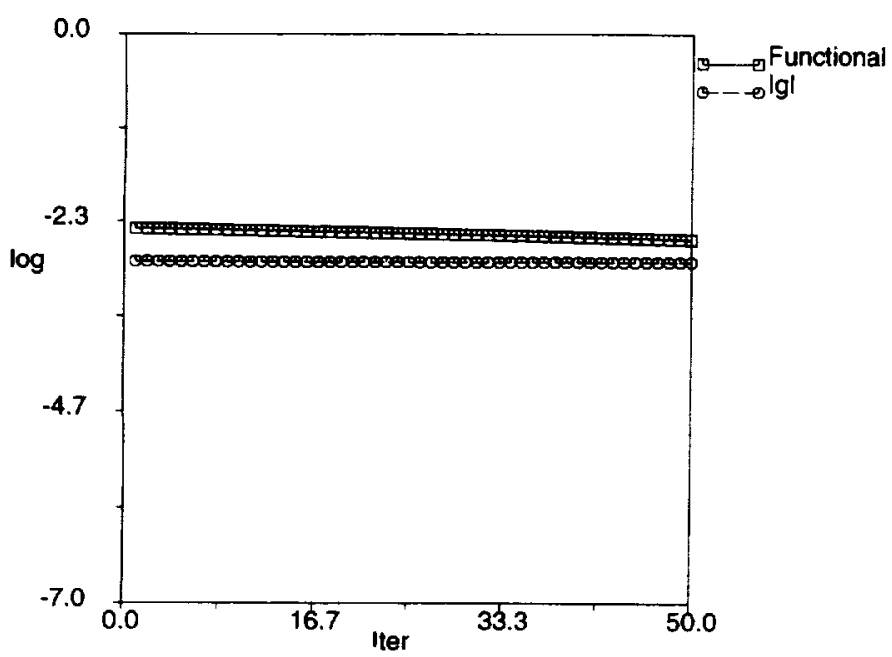

(c) Functional and modulus of gradient versus number of iterations for SD1.

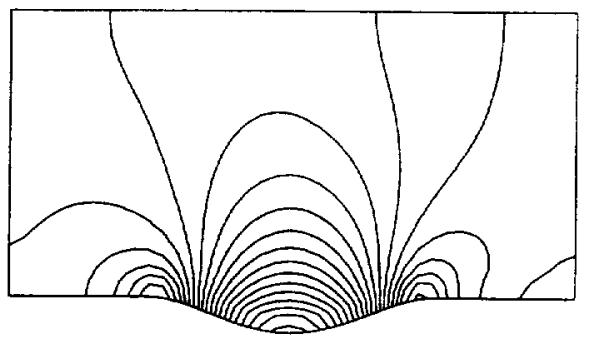

(b) Starting configuration.

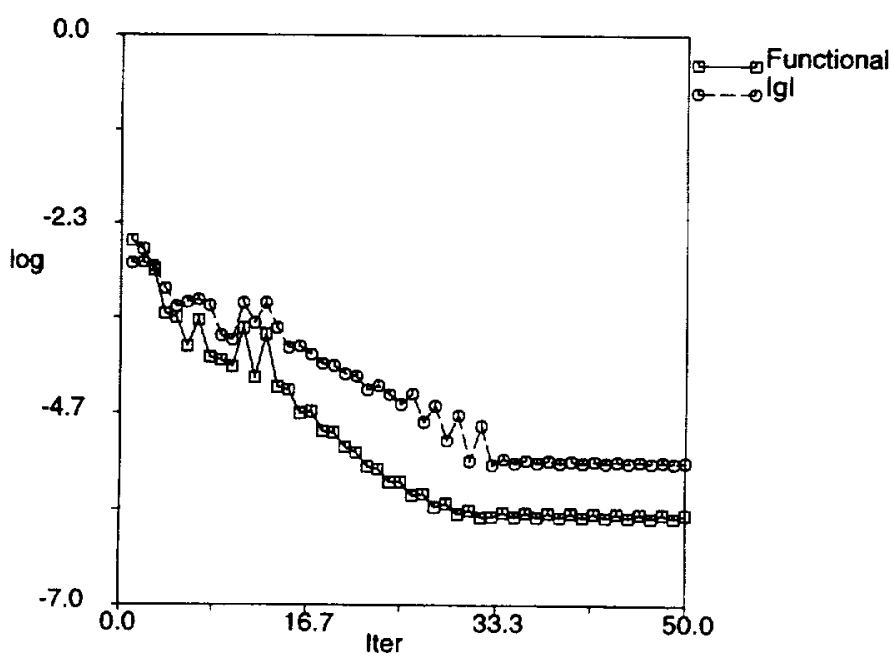

(d) Functional and modulus of gradient versus number of iterations for BFGS2.

Figure 6 


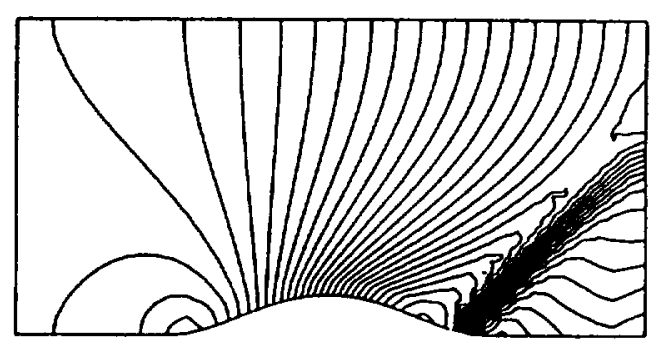

(a) Target Mach number field.

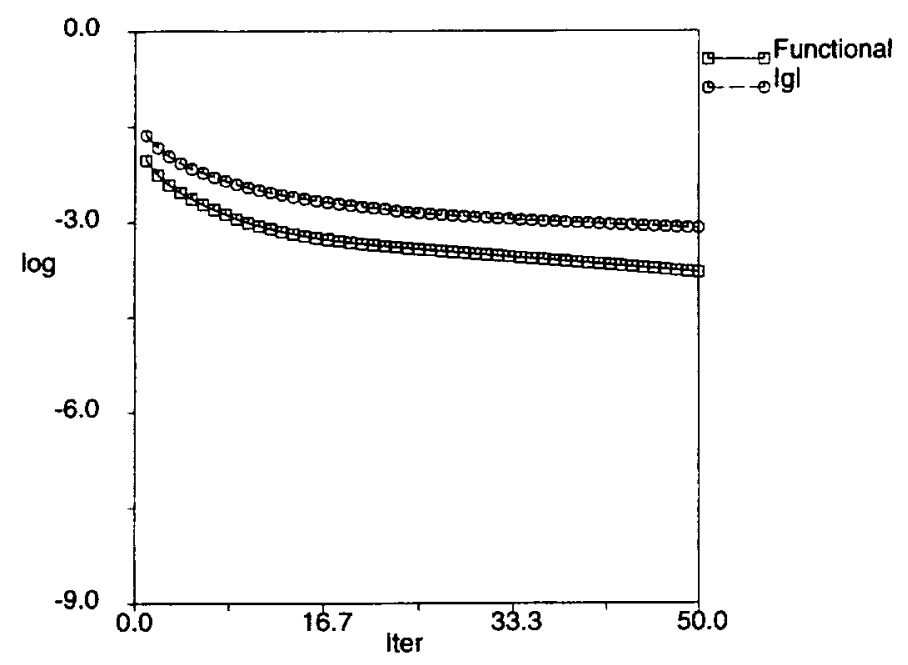

(b) SD1.

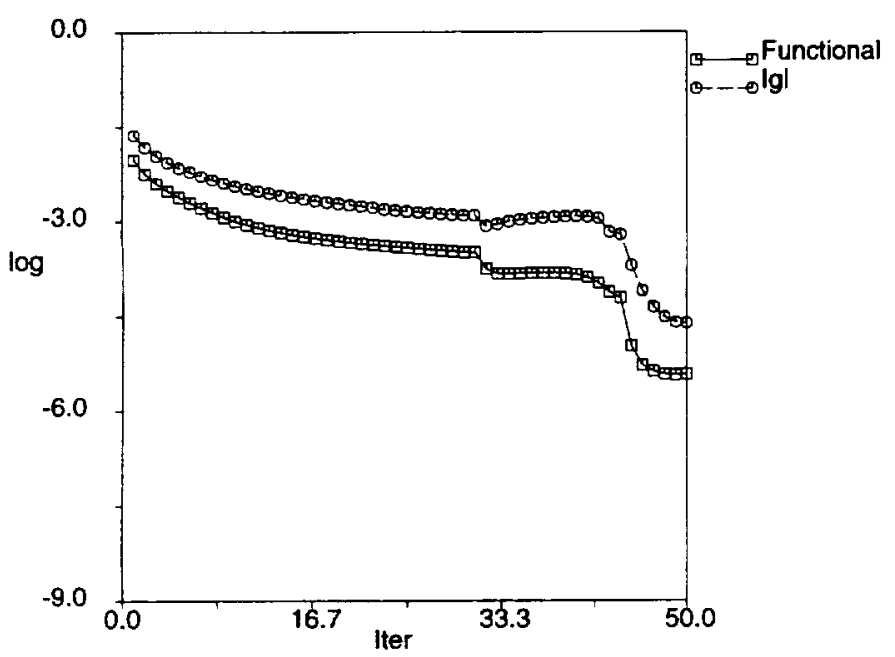

(d) BFGS1.

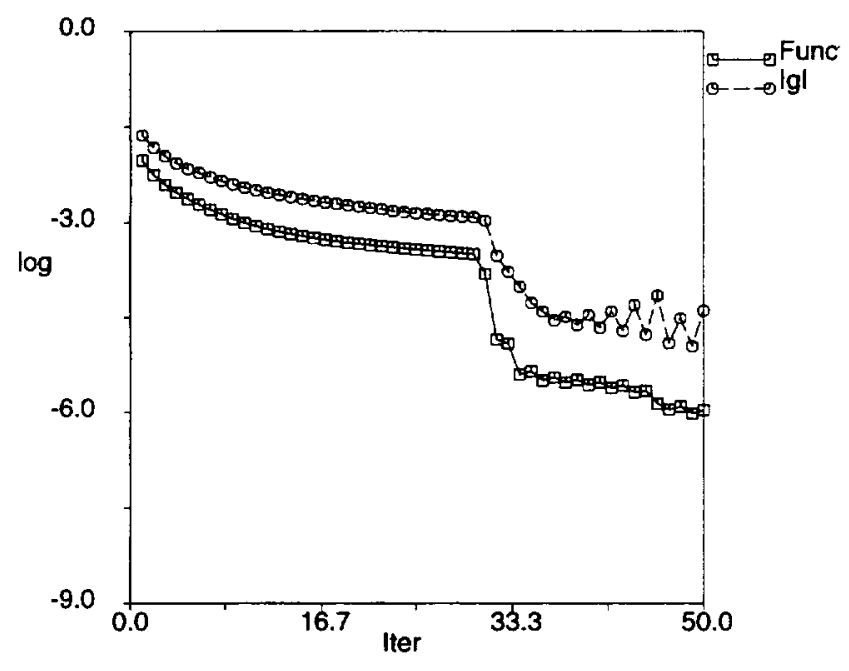

(c) SD2.

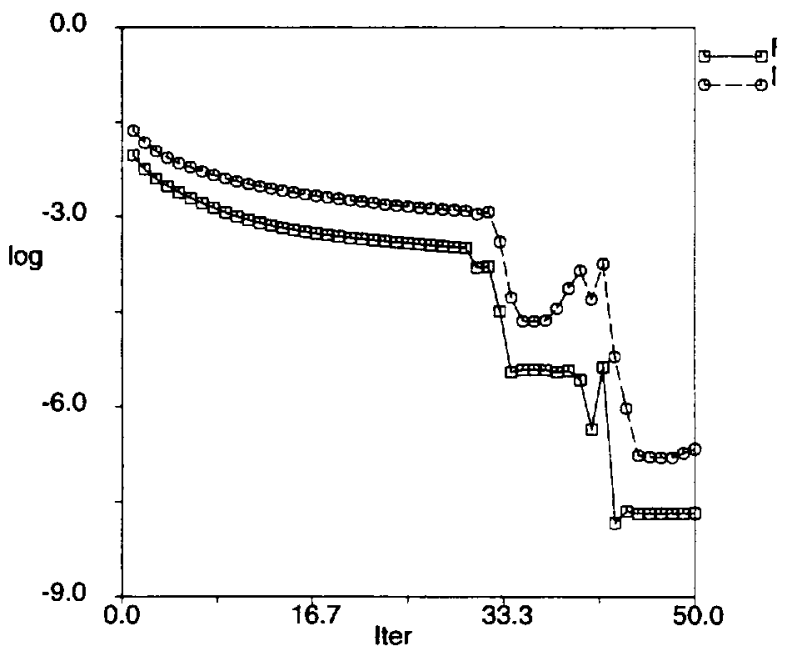

(e) BFGS2.

Figure 7 


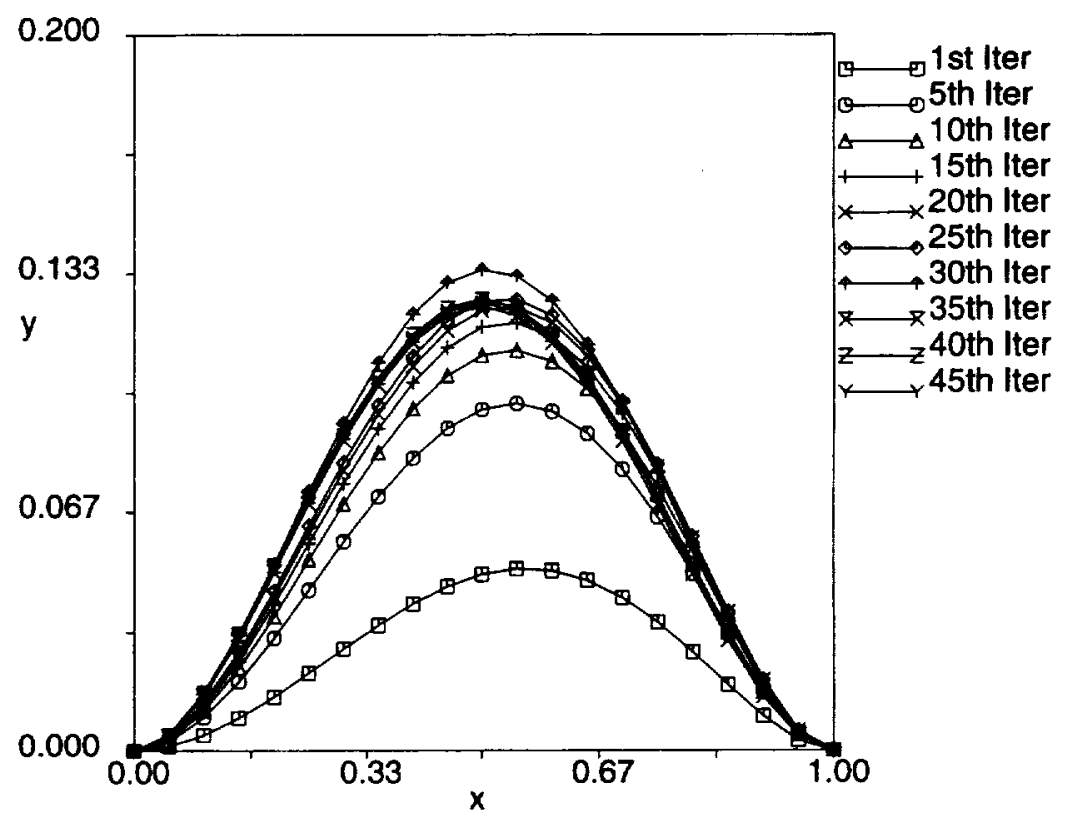

Figure 8: Wall shapes sequence with BFGS2. Starting configuration: constant section. 


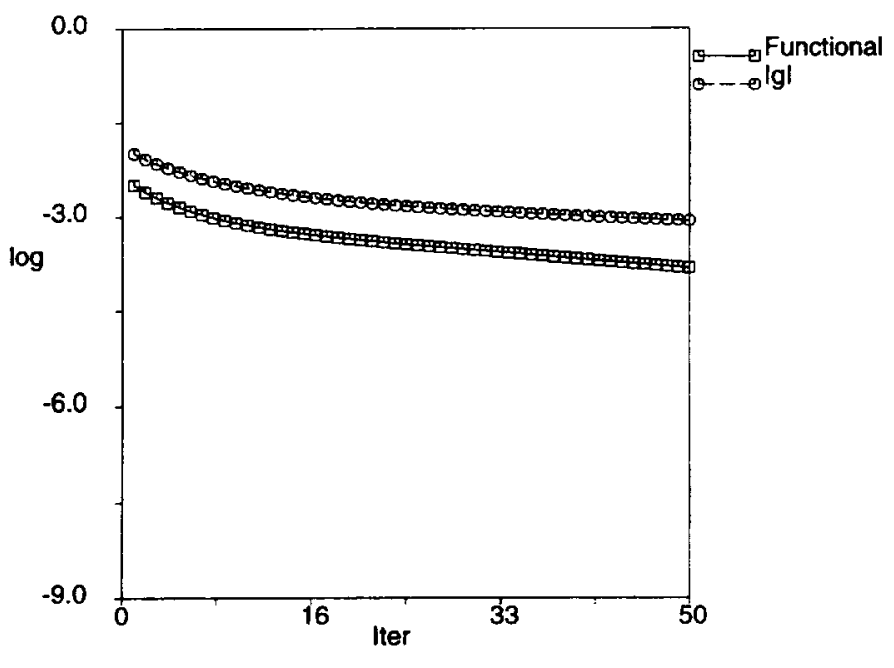

(a) SD1.

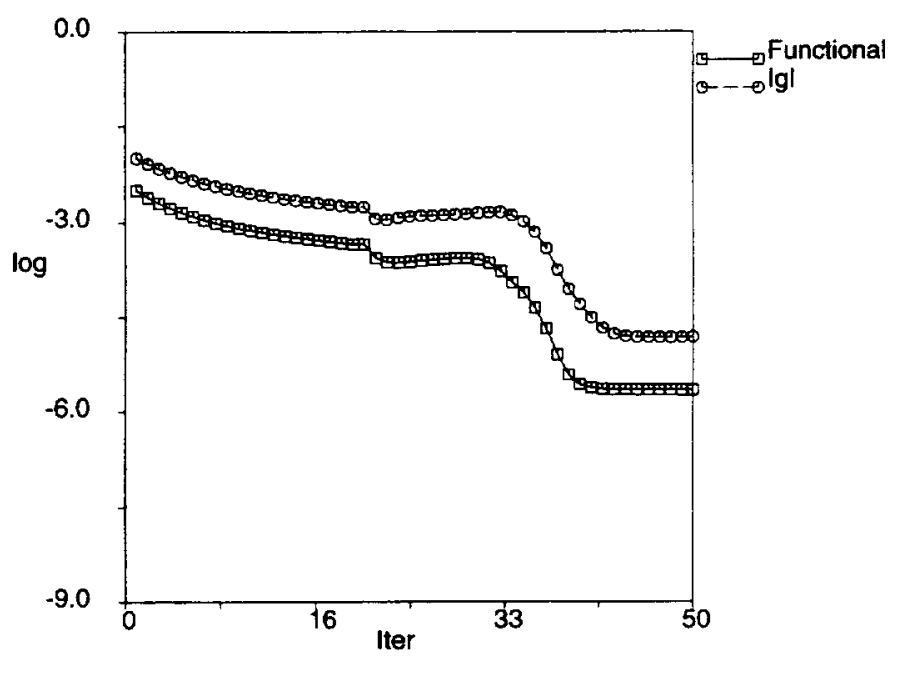

(c) BFGS1.

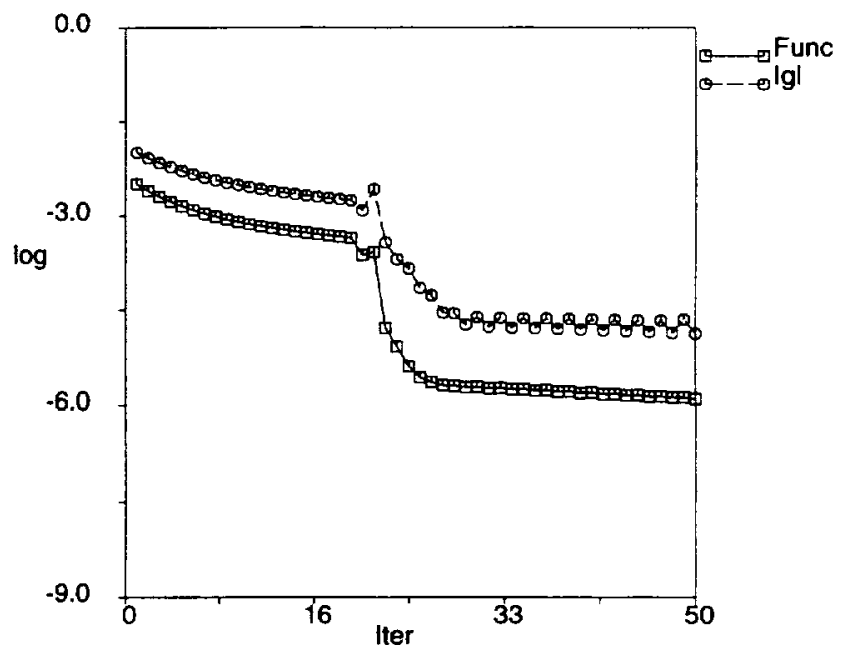

(b) SD2.

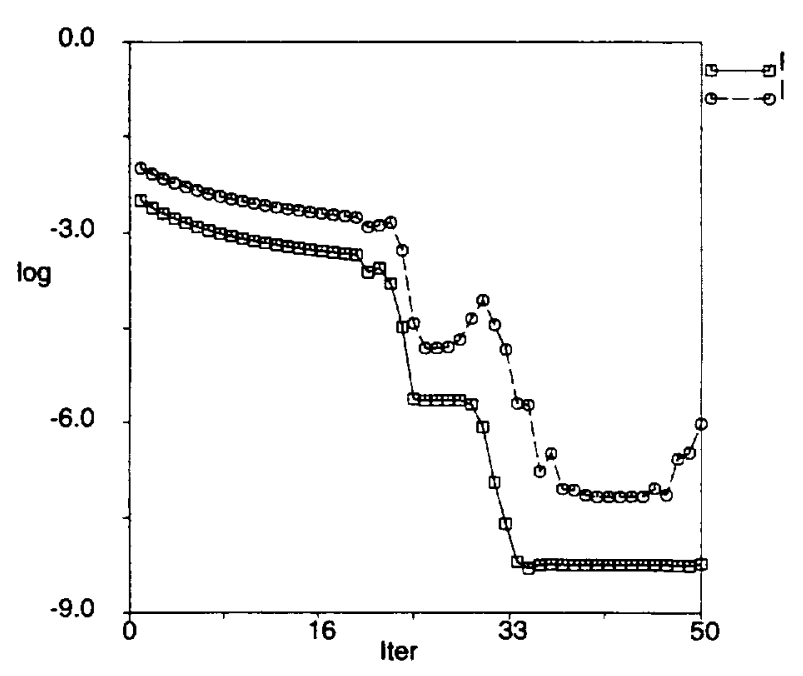

(d) BFGS2.

Figure 9: Results with $20 \times 10$ grid. 


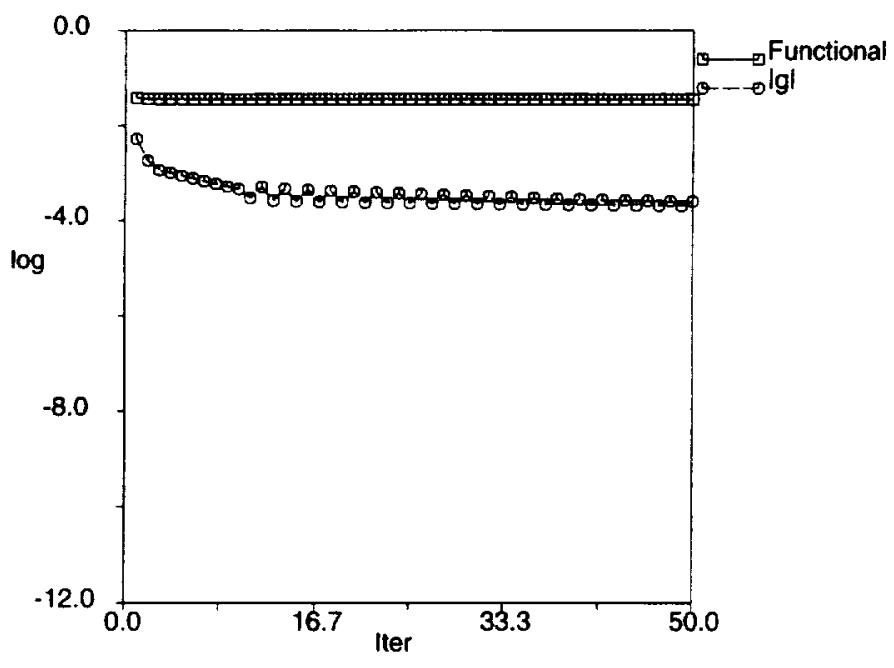

(a) DS2.

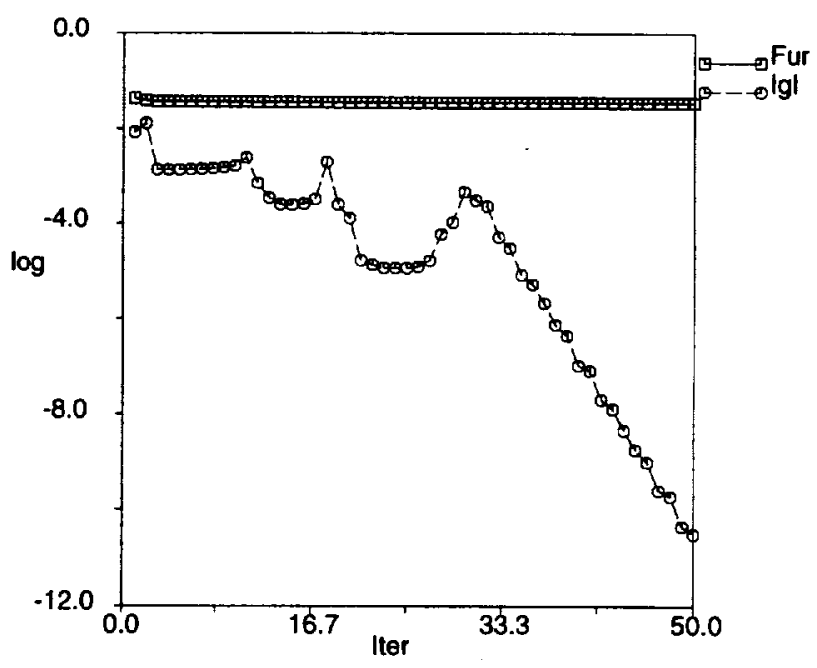

(b) BFGS2.

Figure 10 



\begin{tabular}{|c|c|c|c|}
\hline \multicolumn{3}{|c|}{ REPORT DOCUMENTATION PAGE } & $\begin{array}{l}\text { Form Approved } \\
\text { OMB No. 0704.0188 }\end{array}$ \\
\hline \multicolumn{4}{|c|}{ 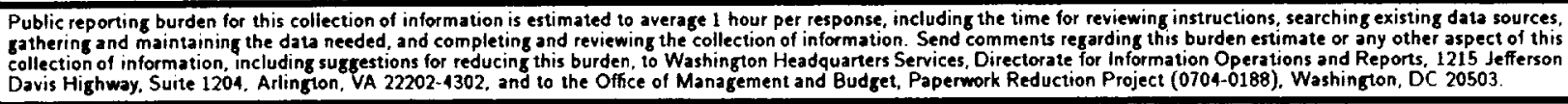 } \\
\hline 1. AGENCY USE ONLY(Leave blank) & $\begin{array}{l}\text { 2. REPORT DATE } \\
\text { March } 1995\end{array}$ & \multicolumn{2}{|c|}{$\begin{array}{l}\text { 3. REPORT TYPE AND DATES COVERED } \\
\text { Contractor Report }\end{array}$} \\
\hline \multicolumn{3}{|c|}{$\begin{array}{l}\text { 4. TITLE AND SUBTITLE } \\
\text { CONTRIBUTION TO THE OPTIMAL SHAPE DESIGN OF TWO- } \\
\text { DIMENSIONAL INTERNAL FLOWS WITH EMBEDDED SHOCKS }\end{array}$} & \multirow[t]{2}{*}{$\begin{array}{l}\text { 5. FUNDING NUMBERS } \\
\text { C NAS1-19480 } \\
\text { WU 505-90-52-01 }\end{array}$} \\
\hline \multicolumn{3}{|l|}{$\begin{array}{l}\text { 6. AUTHOR(S) } \\
\text { Angelo Iollo } \\
\text { Manuel D. Salas }\end{array}$} & \\
\hline \multicolumn{3}{|c|}{$\begin{array}{l}\text { 7. PERFORMING ORGANIZATION NAME(S) AND ADDRESS(ES) } \\
\text { Institute for Computer Applications in Science } \\
\text { and Engineering } \\
\text { Mail Stop 132C, NASA Langley Research Center } \\
\text { Hampton, VA 23681-0001 }\end{array}$} & $\begin{array}{l}\text { RMING ORGANIZATION } \\
\text { T NUMBER } \\
\text { Report No. } 95-20\end{array}$ \\
\hline \multicolumn{3}{|c|}{$\begin{array}{l}\text { 9. SPONSORING/MONITORING AGENCY NAME(S) AND ADDRESS(ES) } \\
\text { National Aeronautics and Space Administration } \\
\text { Langley Research Center } \\
\text { Hampton, VA } 23681-0001\end{array}$} & $\begin{array}{l}\text { 10. SPONSORING/MONITORING } \\
\text { AGENCY REPORT NUMBER } \\
\text { NASA CR-195062 } \\
\text { ICASE Report No. } 95-20\end{array}$ \\
\hline \multicolumn{4}{|c|}{$\begin{array}{l}\text { 11. SUPPLEMENTARY NOTES } \\
\text { Langley Technical Monitor: Dennis M. Bushnell } \\
\text { Final Report } \\
\text { To be submitted to the Journal of Computational Physics }\end{array}$} \\
\hline \multicolumn{2}{|c|}{$\begin{array}{l}\text { 12a. DISTRIBUTION/AVAILABILITY STATEMENT } \\
\text { Unclassified-Unlimited } \\
\text { Subject Category } 64\end{array}$} & \multicolumn{2}{|c|}{ 12b. DISTRIBUTION CODE } \\
\hline \multicolumn{4}{|c|}{$\begin{array}{l}\text { 13. ABSTRACT (Maximum } 200 \text { words) } \\
\text { We explore the praticability of optimal shape design for flows modeled by the Euler equations. We define a functional } \\
\text { whose minimum represents the optimality condition. The gradient of the functional with respect to the geometry } \\
\text { is calculated with the Lagrange multipliers, which are determined by solving a costate equation. The optimization } \\
\text { problem is then examined by comparing the performance of several gradient-based optimization algorithms. In this } \\
\text { formulation, the flow field can be computed to an arbitrary order of accuracy. Finally, some results for internal flows } \\
\text { with embedded shocks are presented, including a case for which the solution to the inverse problem does not belong } \\
\text { to the design space. }\end{array}$} \\
\hline \multirow{2}{*}{\multicolumn{2}{|c|}{$\begin{array}{l}\text { 14. SUBJECT TERMS } \\
\text { Optimal Shape; Internal Flows; Shocks }\end{array}$}} & & $\begin{array}{c}\text { 15. NUMBER OF PAGES } \\
22 \\
\end{array}$ \\
\hline & & & $\begin{array}{c}\text { 16. PRICE CODE } \\
\mathrm{A} 03\end{array}$ \\
\hline $\begin{array}{l}\text { 17. SECURITY CLASSIFICATION } \\
\text { OF REPORT } \\
\text { Unclassified }\end{array}$ & $\begin{array}{l}\text { 18. SECURITY CLASSIFICATION } \\
\text { OF THIS PAGE } \\
\text { Unclassified }\end{array}$ & $\begin{array}{l}\text { 19. SECURITY CLASSIFICATION } \\
\text { OF ABSTRACT }\end{array}$ & $\begin{array}{l}\text { 20. LIMITATION } \\
\text { OF ABSTRACT }\end{array}$ \\
\hline NSN 7540-01-200-5500 & & & $\begin{array}{l}\text { Standard Form 298(Rev. 2-89) } \\
\text { Prescribed by ANSI Sid. Z39-18 } \\
\text { 298-102 }\end{array}$ \\
\hline
\end{tabular}

